\title{
Enhancing Syndromic Surveillance at a Local Public Health Department
}

\author{
Jessica R. White* and Kate Goodin \\ Epidemiology, Maricopa County Department of Public Health, Phoenix, AZ, USA
}

\section{Objective}

To demonstrate how a local public health department used the Centers for Disease Control and Prevention (CDC) Framework for Program Evaluation and a logic model to enhance its syndromic surveillance program.

\section{Introduction}

The mission of the Maricopa County Department of Public Health (MCDPH; Arizona) is to protect and promote the health and well-being of its residents and visitors. Surveillance efforts allow epidemiologists to quantify and characterize public health threats, but traditional methods take time. In an effort to enhance situational awareness, the Office of Epidemiology dedicated resources to begin developing a robust syndromic surveillance program. This abstract outlines steps for enhancing syndromic surveillance at a local public health department.

\section{Methods}

The CDC Framework for Program Evaluation was used to systematically improve MCDPH's existing syndromic surveillance program. [1] First, stakeholders from the state and county syndromic surveillance programs were engaged. The MCDPH Syndromic Surveillance Strategic Planning and Development Workgroup was formed to identify existing resources, current challenges, and a unified mission. Meetings were arranged with Arizona Department of Health Services (ADHS) staff to exchange ideas for future projects. Second, a logic model was created to describe MCDPH's existing and future syndromic surveillance efforts. The MCDPH logic model was influenced by the National Syndromic Surveillance Program's logic model for enhancing syndromic surveillance capacity and practice. [2] Third, the scope of the program was focused by identifying five priority initiatives for the year. The remaining steps are in progress. Plans were established to measure outcomes of the program, evaluate progress for meeting goals, and share lessons learned.

\section{Results}

The MCDPH Strategic Planning Workgroup has been meeting bi-weekly since June 2015. The Workgroup identified goals and activities and organized them in a logic model (Figure). Using input from state and county public health officials, five priority syndromes were identified: heat-related illness, arboviral disease, drug overdose, influenza-like illness, and gastrointestinal illness. For each syndrome, Workgroup members began (1) developing technical guides for accessing and analyzing data, and (2) seeking collaborations with external entities. MCDPH is now actively involved with the following activities: CSTE Heat Syndrome Workgroup, ADHS Arboviral Syndromic Surveillance Use Case Project, and "Flu Near You" local use initiative. The Workgroup plans to review its progress quarterly and adjust activities that are not adequately achieving goals. By sharing MCDPH's experience, the Workgroup is achieving a goal to contribute knowledge to the nation-wide community of practice.

\section{Conclusions}

In MCDPH's experience, the CDC Framework for Program Evaluation was an effective tool for strategic planning, while the logic model helped focus efforts on the appropriate initiatives. In less than 3 months, the Workgroup collaborated with local, state, and national stakeholders, identified challenges faced by the existing program, prioritized goals, and launched activities to enhance surveillance for five priority syndromes. The immediate next steps will be to finalize technical guides, validate syndromic surveillance queries, evaluate progress of the program, and continue to share lessons learned with the community of practice. The authors hope that by sharing this experience, other public health practitioners will be encouraged to enhance syndromic surveillance at their local health departments.

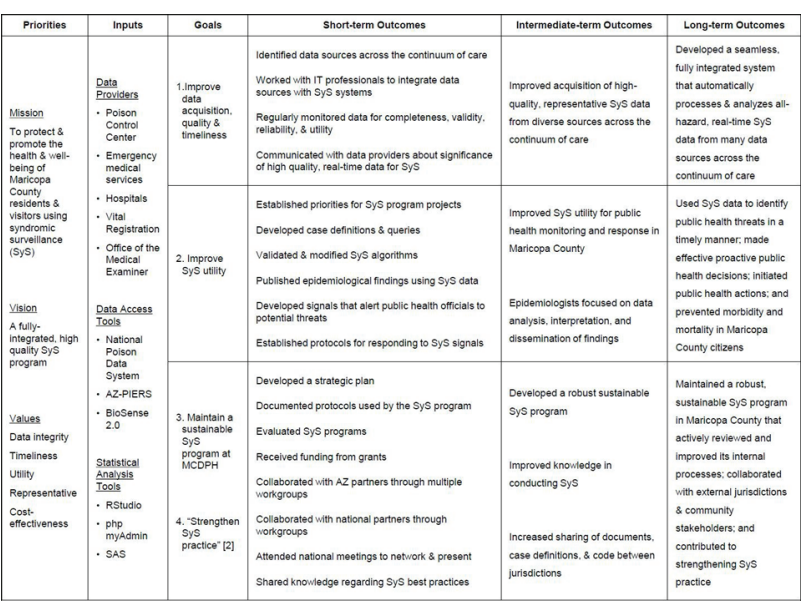

Logic Model of the Syndromic Surveillance Program at the Maricopa County Department of Public Health

\section{Keywords}

Strategic planning; Evaluation; Local level

\section{Acknowledgments}

The authors thank the MCDPH Syndromic Surveillance Strategic Planning Workgroup for its contributions.

\section{References}

1. Centers for Disease Control and Prevention. Framework for program evaluation in public health. MMWR 1999;48(No. RR-11).

2. Centers for Disease Control and Prevention. National Syndromic Surveillance Program Funding Opportunity Announcement Logic Model [Internet]. Atlanta (GA): CDC Division of Health Informatics and Surveillance; 2015 Mar 20 [cited 2015 Aug 31]. Available from: http://www.cdc.gov/nssp/foa-logic-model.html.

\footnotetext{
*Jessica R. White

E-mail: JessicaWhite@mail.maricopa.gov
} 